\title{
Manejo clínico de la equinococosis quística hepática, estadio CE3b, combinando tratamiento médico-estrategia de vigilancia y espera: a propósito de un caso
}

Daniel Da Rosa*, Ana María Acuña*, Elisa Figueredo*, Luis Calegari ${ }^{*}$ Luis Ruso Martínez ${ }^{\dagger}$

\section{Resumen}

La hidatidosis o equinococosis quística es una enfermedad zoonótica que tiene presentaciones clínicas muy heterogéneas. Los quistes sintomáticos y complicados habitualmente son resueltos mediante cirugía siguiendo las reglas de la WHO-IWGE. Sin embargo, en las formas transicionales (CE3) los criterios terapéuticos continúan en discusión. Asimismo, en quistes asintomáticos y en pacientes con alto riesgo quirúrgico, la opción del tratamiento médico con benzoimidazólicos y su combinación con la estrategia de vigilar y esperar puede ser una alternativa terapéutica segura. Se presenta un caso de hidatidosis hepática CE3b con características clínico-evolutivas excepcionales y alto riesgo quirúrgico en que se adoptó un manejo de vigilancia clínica y tratamiento con albendazol. Se analizan los beneficios y riesgos de esta terapéutica.

Palabras clave: EQUINOCOCOCIS HEPÁTICA BENZOIMIDAZOLES VIGILANCIA

Key words: HEPATIC ECHINOCOCCOSIS BENZIMIDAZOLES SURVEILLANCE

\section{Introducción}

La equinococosis quística o hidatidosis es una enfermedad zoonótica causada por el parasitismo de la larva de Echinococcus granulosus, que continúa siendo un grave problema sanitario en el mundo, en la región y en Uruguay $^{(1)}$.

El hígado es la localización más frecuente; según nuestra casuística representa el 59\% de los casos, con una gran variabilidad en su presentación clínica y sintomatología ${ }^{(2)}$.

El diagnóstico se basa en la presentación clínica, factores de riesgo epidemiológicos, imagenología y serología, teniendo esta última un rol complementario ${ }^{(3)}$.

Actualmente los criterios generales de tratamiento se ajustan a los criterios establecidos a partir de la clasificación WHO-CE ${ }^{(4)}$ y la clásica de Gharbi ${ }^{(5)}$, los que dependen de la localización, tamaño, presencia de complicaciones, quistes y riesgos de diseminación. Sin embargo, existe una población de casos asintomáticos y no complicados en los cuales se discuten los resultados de la cirugía (abierta o laparoscópica) frente a otras alternativas de tratamiento médico, percutánea o una estrategia de vigilancia y espera, o ambas ${ }^{(6,7)}$.

En algunos casos la combinación de terapéutica médica con albendazol (ABZ) en series ( 3 a 6 ) de 30 días con descanso de 15 días entre ellos es recomendada, continuando con un seguimiento regular por imagenología anual ${ }^{(6-8)}$.

El propósito de este trabajo es presentar un caso de equinococosis quística hepática $\mathrm{CE} 3 \mathrm{~b}$, con alto riesgo quirúrgico, tratado mediante vigilancia/esperar y alben-

\footnotetext{
* Departamento de Parasitología y Micología, Instituto de Higiene, Facultad de Medicina, Universidad de la República, Uruguay † Clínica Quirúrgica 3, Hospital Maciel, Facultad de Medicina, Universidad de la República, Uruguay. Recibido: 12/6/17

Aprobado: 30/10/17
} 
dazol, y analizar los riesgos y beneficios de la terapéutica adoptada.

\section{Caso clínico}

Hombre de 81 años, sexo masculino, sin antecedentes patológicos a destacar. Índice de Karnofsky 100. Asintomático, procedente de medio rural, portador de múltiples imágenes con patrón imagenológico de hidatidosis hepática en lóbulo derecho, conocida desde hace 30 años. No tiene estudios imagenológicos, ni tratamientos previos a la tomografía axial computada (TAC) realizada en 2013, en la cual se observa, en segmento VII, imagen quística de 62 por $60 \mathrm{~mm}$ de aspecto polilobulado con un sector redondeado intrahepático de $6,5 \mathrm{~cm}$ con calcificaciones periféricas y vesículas hijas en su interior y varias formaciones contiguas de similares características emergentes del hígado, la mayor de $55 \mathrm{~mm}$, además de otra más pequeña de $20 \mathrm{~mm}$, catalogada como quiste hidático estadio CE3b. En segmento VIII se observa otra imagen de $7 \mathrm{~mm}$, estadio CE1. Paraclínica: hemograma y función hepático y renal normales.

Consulta con cirujano, se considera que se trata de una hidatidosis hepática múltiple en diferentes estadios evolutivos, con predominio de la forma CE3b y evasión extrahepática posterior, con riesgos evolutivos de rotura a peritoneo y potencial siembra considerando la estadificación "transicional" de los quistes y de tránsito transdiafragmático hacia el tórax por la localización de los quistes posterosuperiores. Se estiman más alejados los riesgos de apertura en vías biliares y en sistema venoso cavo-suprahepático. Asimismo, se evalúan los riesgos quirúrgicos propios de la topografía de los quistes, así como de graves complicaciones intraoperatorias (hemorragia, diseminación) y posoperatorias de enfermedad de la adventicia hidática vinculada a las áreas calcificadas de la misma y las limitantes para realizar una quistoadventicectomía total, así como la morbilidad de una incisión abdominal amplia y que incluso puede requerir asociar un abordaje torácico simultáneo (toraco-freno laparotomía). Esta situación clínico-patológica se da en el contexto de un paciente totalmente asintomático durante 30 años y con calidad de vida normal a los 81 años. Explicada y comprendida la situación por el paciente, se sugiere control evolutivo (wacht and wait) con tratamiento médico asociado.

Se realizó tratamiento médico en 2014: tres series de ABZ $(15 \mathrm{mg} / \mathrm{kg} /$ día - dosis total repartida en dos comidas con alto contenido de tenor graso para la mejor absorción del fármaco-, cada serie fue de 30 días con 15 días de descanso entre las mismas). Se efectuaron controles paraclínicos, hemograma, funcional hepático y renal de base y cada 15 días durante todo el tratamiento, sin presentar efectos secundarios. Se resuelve la realiza- ción de estudio imagenológico en 2015, a un año luego de finalizado el tratamiento.

En la TAC de 2015 no se observaron cambios en los quistes en segmento VII, tanto en forma como en tamaño, presentando discreto aumento de las calcificaciones de la pared quística. En cuanto a su contenido, este es más homogéneo y no se visualizan las estructuras internas (vesículas hijas) observadas en estudio imagenológico anterior al tratamiento médico (figura 1). Se recomienda continuar con control clínico-imagenológico anual, resultando asintomático a 33 meses de seguimiento.

\section{Consideraciones y recomendaciones}

La equinococosis quística o hidatidosis (EQ) es una enfermedad que se caracteriza por presentaciones clínicas heterogéneas. En los últimos diez años, en nuestra experiencia, las presentaciones clínicas con complicaciones sintomáticas han venido decreciendo. La introducción de la clasificación según aspectos imagenológicos (WHO-IGWE), seguido por el consenso de expertos del año $2010^{(6)}$, ha proporcionado un marco adecuado para la consideración del manejo clínico de pacientes asintomáticos con quistes en estadio transicional CE3. Sin embargo, estas consideraciones están basadas en evidencia clínica derivada de consensos y de trabajos de limitada calidad ${ }^{(7)}$.

Cuatro son las modalidades de tratamiento actualmente aceptadas en la EQ: cirugía (abierta o laparoscópica), tratamiento percutáneo, tratamiento médico con derivados benzoimidazólicos (albendazol [ABZ] o praziquantel [PZQ], o ambos) y un último enfoque de vigilancia y espera (watch and wait) ${ }^{(8)}$. Sin embargo, se carece de estudios longitudinales controlados que comparen la eficacia y la efectividad de las diferentes opciones de tratamiento, sean esta solas o combinadas y su aplicación en cada etapa clínica específica. En consecuencia, cuál podrá ser la mejor opción para el tratamiento de la EQ de hígado es una pregunta que presenta aristas aún controversiales.

Uno de los puntos a considerar es la localización en el órgano. Se ha considerado que los quistes ubicados en sectores superficiales y accesibles, sean sintomáticos o asintomáticos, podrían tener una resolución quirúrgica. Esta propuesta incluye un riesgo operatorio que aun en cirugías no complicadas se estima entre $5 \%$ y $10 \%{ }^{(9)}$. También debe discutirse el valor, su tamaño y estadio. Diferentes series ubican el tamaño máximo para considerar una respuesta positiva al tratamiento médico en quistes menores de $5 \mathrm{~cm}^{(10,11)}$. En nuestro medio hemos tenido buena respuesta en un rango de mayor tamaño, considerando aquellos menores o iguales a $7 \mathrm{~cm}^{(2)}$. 


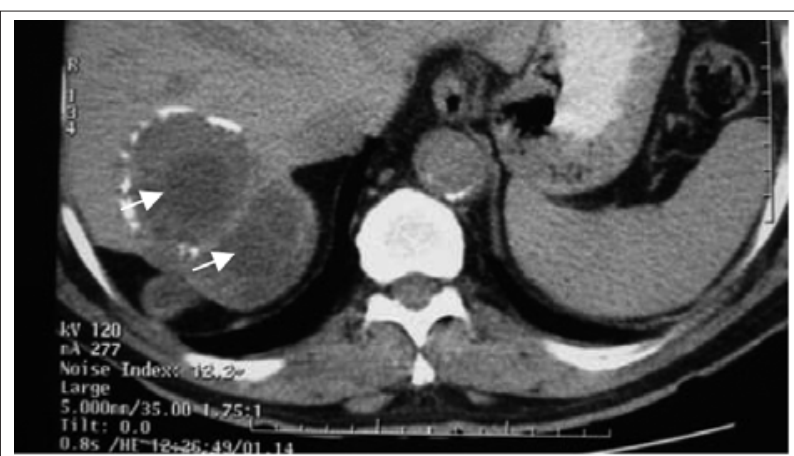

A

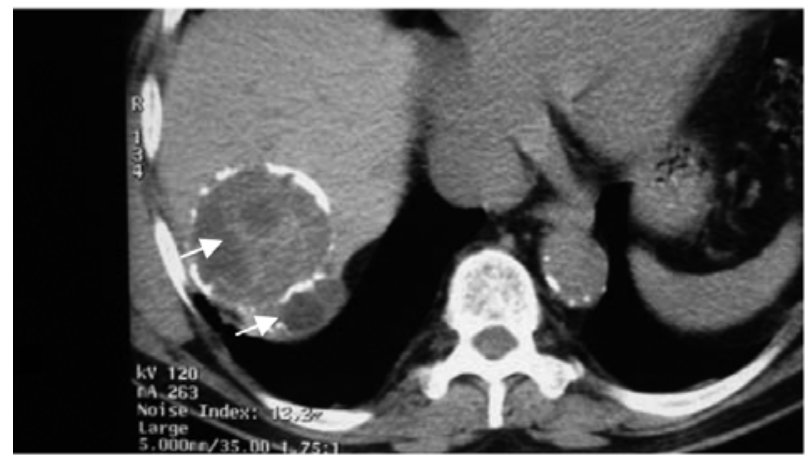

A

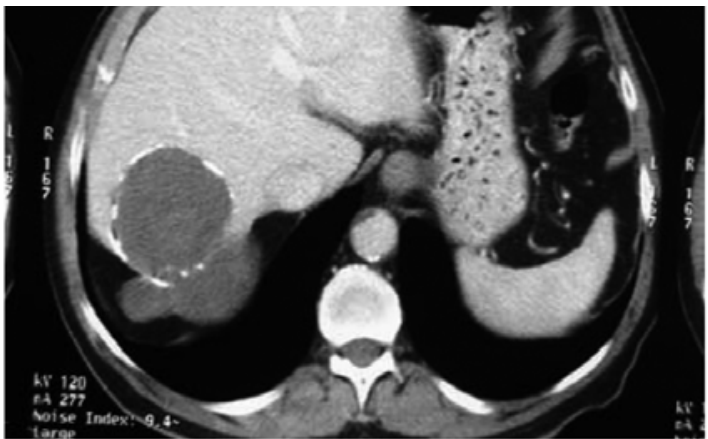

B

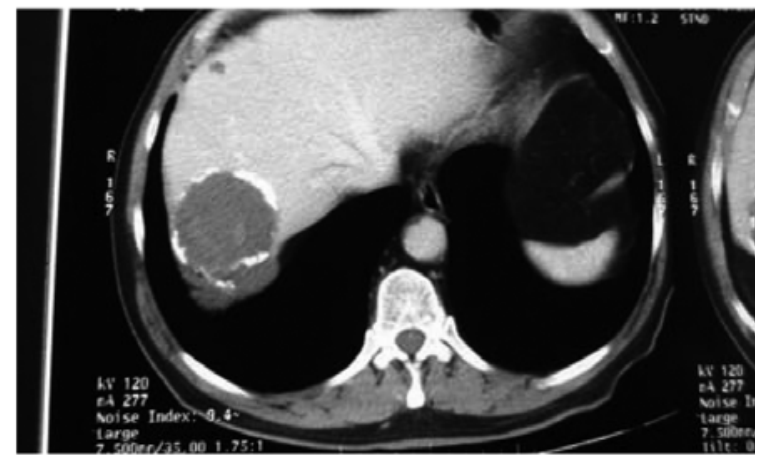

B

Figura 1. Tomografía computada axial. A: antes de iniciar el tratamiento (2013), EC3b con múltiples imágenes compatibles a vesículas hijas en el interior del quiste (flechas). B: (2015) desaparición de los elementos activos y disminución del tamaño quístico al año del tratamiento médico con ABZ, pasando a un estadio inactivo EC3a.

Por otra parte, el definir el estadio del quiste por imagenología es una consideración ineludible para considerar la resolución del paciente. Clásicamente se ha utilizado la clasificación de Gharbi, pero paulatinamente la clasificación de la WHO-IGWE está siendo más usada. En esta se establece que los estadios CE1 a CE2 presentan la condición de ser viables. En este sentido, cabe la consideración de que los quistes en estadio CE3 son quistes en "transición" y se dividen en estadio CE3a, el cual puede ser activo o inactivo, y CE3b, que se considera biológicamente activo. Siendo los estadios C4 a C5 quistes inactivos, lo que nos hace comprender la historia natural del quiste hidático ${ }^{(7,10)}$.

Otro aspecto a considerar tiene que ver con las dosis y el tiempo de tratamiento con los derivados benzoimidazólicos: en Uruguay se realizan terapéuticas con ABZ o PZQ, o ambos, a dosis de $15 \mathrm{mg}$ y $40 \mathrm{mg} / \mathrm{kg} /$ día respectivamente, de 3 a 6 series, como fuera expresado anteriormente $^{(2)}$. Sin embargo, en la provincia de Río Negro, Argentina, suelen realizarse tratamientos continuos durante 90 días a dosis de $10 \mathrm{mg} / \mathrm{kg} /$ día $^{(12,13)}$. Aun así, algunos autores consideran que los estadios CE3b tienen menor respuesta al tratamiento no quirúrgico y la recaí- da ocurre, casi invariablemente, enseguida que se discontinúa el albendazol ${ }^{(14,15)}$.

La mejor estrategia para los quistes CE3b no está definida. El tiempo de inactivación de quistes estadios $\mathrm{C} 3 \mathrm{~b}$ ha sido estudiado y se ha informado una rápida y sostenida inactividad con el tratamiento médico con ABZ en series (tiempo de 4 a 9,5 meses), con un porcentaje de recaídas menor a 10\%, con imagenología incambiada en un $50 \%$. Las variables que inciden directamente en las recaídas o activaciones de quistes CE3 fueron identificadas por Rinaldi ${ }^{(8)}$ en la misma serie de pacientes: interrupción del tratamiento, pérdida en el seguimiento o desafiliación al tratamiento por parte del paciente considerando su estado asintomático.

En el seguimiento imagenológico de los quistes hepáticos la ultrasonografía o ecografía es un método imagenológico adecuado por su bajo costo. Sin embargo, al ser técnico dependiente, es posiblemente discutida su utilidad. En nuestro medio, la alta especialización de los técnicos que la realizan hace que debamos recomendar este método para el seguimiento cada seis meses y realizar TAC una vez al año. En otras localizaciones es posible que sea recomendable realizar TAC o resonancia nu- 
clear magnética anual. En todos los casos el seguimiento imagenológico debería realizarse por períodos amplios, de por lo menos tres a cinco años ${ }^{(2,16,15)}$.

De hecho, Stojkovic ${ }^{(18)}$ recomienda un seguimiento de cinco años para confirmar la estabilidad de un quiste calificado como inactivo.

Los estudios inmunológicos han tenido poca utilidad en el seguimiento de estos pacientes; sin embargo, en los últimos años se ha propuesto la utilización de recombinantes del $\mathrm{AgB}$ con resultados prometedores ${ }^{(19,20)}$.

El presente caso es un ejemplo que verifica el valor del inicio precoz del tratamiento médico, una vez evaluado íntegramente al paciente desde una perspectiva de costo-beneficio terapéutico frente a los riesgos de complicaciones asociados a la cirugía.

La utilización de la estrategia de vigilancia y espera resulta beneficiosa no solo en los casos sintomáticos con alto riesgo quirúrgico, sino en aquellos casos asintomáticos. Stojkovic demuestra, mediante un estudio retrospectivo de quistes hidáticos inactivos, el empleo con buenos resultados de esta estrategia y propone el seguimiento mínimo de tres años con un margen de seguridad de seguimiento a cinco años. Observa que el período de reactivación de los quistes hidáticos en su estudio es inferior a los 18 meses $^{(18)}$.

En base a los conocimientos actuales es factible la utilización de la estrategia de vigilancia y espera en pacientes asintomáticos con quistes catalogados como estadios CE3 (CE3a o CE3b) con tratamiento médico combinado. En casos con alto riesgo quirúrgico esta conducta - por lo menos a mediano plazo- parece ser una alternativa segura y recomendable. Sin embargo, es necesario realizar estudios clínicos con mayor nivel de evidencia que permitan eliminar las actuales controversias y asegurar una estrategia terapéutica segura y curativa.

\section{Abstract}

Cystic echinococcosis is a zoonotic disease with very heterogeneous clinical presentations. The possibility of symptomatic hepatic cysts be solved by surgery is the conduct of election. However, in asymptomatic cysts and in patients with high surgical risk, medical treatment with benzimidazolic combined with the strategy of watch and wait, may be an option to take into account. In the present work, we take as an example a case of hepatic hydatid disease with high surgical risk and analyze the potential benefits of establishing such therapeutic conduct and monitoring.

\section{Resumo}

A hidatidose ou equinococose cística é uma doença zoonótica com apresentações clínicas muito heterogê- neas. Os cistos sintomáticos e complicados de maneira geral são tratados por cirurgia de acordo com as indicações da WHO- IWGE. No entanto, nos casos de formas transicionais (CE3) os critérios terapêuticos continuam em discussão. Nos cistos assintomáticos e em pacientes com alto risco cirúrgico a opção do tratamento médico com benzoimidazolicos e sua combinação com a estratégia de vigilar e esperar, pode ser uma alternativa terapêutica segura. Apresenta-se um caso de hidatidose hepática CE3b, com características clínico-evolutivas excepcionais e alto risco cirúrgico, no qual utilizou-se um manejo de vigilância clínica e tratamento com albendazol. Os benefícios e os riscos desta terapêutica são analisados.

\section{Bibliografía}

1. Craig PS, McManus DP, Lightowlers MW, Chabalgoity JA, Garcia HH, Gavidia CM, et al. Prevention and control of cystic echinococcosis. Lancet Infect Dis 2007; 7(6):385-94.

2. Bonifacino R, Carter SD, Craig PS, Almeida I, Da Rosa D. Assessment of the immunological surveillance value of humoral and lymphocyte assays in severe human cystic echinococcosis. Trans R Soc Trop Med Hyg 2000; 94(1):97-102.

3. Brunetti E, Junghanss T. Update on cystic hydatid disease. Curr Opin Infect Dis 2009; 22(5):497-502.

4. World Health Organization. Department on Communicable Disease, Surveillance and Response. Puncture, aspiration, injection, re-aspiration: an option for the treatment of cystic echinococcosis. Document WHO/CDS/CSR/APH/2001.6. Geneva: WHO, 2001.

5. Gharbi HA, Hassine W, Brauner MW, Dupuch K. Ultrasound examination of the hydatic liver. Radiology 1981; 139(2):459-63.

6. Brunetti E, Kern P, Vuitton DA; Writing Panel for the WHO-IWGE. Expert consensus for the diagnosis and treatment of cystic and alveolar echinococcosis in humans. Acta Trop 2010; 114(1):1-16.

7. World Health Organization. Informal Working Group. International classification of ultrasound images in cystic echinococcosis for application in clinical and field epidemiological settings. Acta Trop 2003; 85(2):253-61.

8. Rinaldi F, De Silvestri A, Tamarozzi F, Cattaneo F, Lissandrin R, Brunetti E. Medical treatment versus "Watch and Wait" in the clinical management of CE3b echinococcal cysts of the liver. BMC Infect Dis 2014; 14:492.

9. Daradkeh S, El-Muhtaseb H, Farah G, Sroujieh AS, Abu-Khalaf M. Predictors of morbidity and mortality in the surgical management of hydatid cyst of the liver. Langenbecks Arch Surg 2007; 392(1):35-9.

10. Piccoli L, Tamarozzi F, Cattaneo F, Mariconti M, Filice C, Bruno A, et al. Long-term sonographic and serological follow-up of inactive echinococcal cysts of the liver: hints for 
a "watch-and-wait" approach. PLoS Negl Trop Dis 2014; 8(8):e3057.

11. Stojkovic M, Zwahlen M, Teggi A, Vutova K, Cretu CM, Virdone R, et al. Treatment response of cystic echinococcosis to benzimidazoles: a systematic review. PLoS Negl Trop Dis 2009; 3(9):e524.

12. Larrieu E, Del Carpio M, Salvitti JC, Mercapide C, Sustersic J, Panomarenko H, et al. Ultrasonographic diagnosis and medical treatment of human cystic echinococcosis in asymptomatic school age carriers: 5 years of follow-up. Acta Trop 2004; 91(1):5-13.

13. Larrieu E, Frider B, del Carpio M, Salvitti JC, Mercapide C, Pereyra R, et al. Portadores asintomáticos de hidatidosis: epidemiología, diagnóstico y tratamiento. Rev Panam Salud Pública 2000; 8(4):250-6.

14. Kabaalioðlu A, Ceken K, Alimoglu E, Apaydin A. Percutaneous imaging-guided treatment of hydatid liver cysts: do long-term results make it a first choice? Eur J Radiol 2006; 59(1):65-73.

15. Franchi C, Di Vico B, Teggi A. Long-term evaluation of patients with hydatidosis treated with benzimidazole carbamates. Clin Infect Dis 1999; 29(2):304-9.
16. Junghanss T, da Silva AM, Horton J, Chiodini PL, Brunetti E. Clinical management of cystic echinococcosis: state of the art, problems, and perspectives. Am J Trop Med Hyg 2008; 79(3):301-11.

17. Rinaldi F, Brunetti E, Neumayr A, Maestri M, Goblirsch S, Tamarozzi F. Cystic echinococcosis of the liver: A primer for hepatologists. World J Hepatol 2014; 6(5):293-305.

18. Stojkovic M, Rosenberger KD, Steudle F, Junghanss T. Watch and Wait management of inactive cystic echinococcosis, does the path to inactivity matter: analysis of a prospective patient cohort. PLoS Negl Trop Dis 2016; 10(12): e0005243.

19. Vuitton DA, Wen H. Treatment of cystic echinococcosis: a combination of general goals and rules, individual decisions and indications. Neth J Med 2007; 65(3):86-8.

20. Manzano-Román R, Sánchez-Ovejero C, Hernández-González A, Casulli A, Siles-Lucas M. Serological diagnosis and follow-up of human cystic echinococcosis: a new hope for the future? Biomed Res Int 2015; 2015:428205. 